\title{
GRUSHKO-LIKE THEOREMS FOR A CLASS OF FINITELY PRESENTED SEMIGROUPS
}

\author{
ANN YASUHARA
}

\begin{abstract}
An analogue of Grushko's theorem and the unsolvability of the isomorphism problem hold for semigroups in the class of all finitely presented semigroups that have a presentation in which there is no defining relation of the form $1 \Leftrightarrow A$; this class is not recursive but is recursively enumerable.
\end{abstract}

We will define a class of finitely presented semigroups in terms of combinatorial properties of their presentations. For that class we prove (Theorem 1) theorems similar to Grushko's theorem for finitely presented groups (see, for example, [K, p. 58]), and (Theorem 2) a theorem similar to a theorem of Rabin (see [R, Theorem 3.1]), namely, for any given semigroup in that class there is no algorithm to decide of each finitely presented semigroup whether or not it is isomorphic to the given semigroup. We also show (Theorem 3 ) that the class is not recursive.

We assume that the reader is familiar with the standard notions and terminology (as in [D] or [Y1]) of Thue systems, finitely presented semigroups, free products of semigroups, solvable and unsolvable problems and partial recursive functions. We will be thinking of a Thue system, denoted by $T$, as a finite presentation of a semigroup and denote the semigroup presented by $T$ by $S_{T}$. In general, let $T=\left\langle a_{1}, \cdots, a_{m} ; A_{i} \Leftrightarrow A_{i}^{\prime}\right.$, $1 \leqq i \leqq n\rangle$ where $\left\{a_{1}, \cdots, a_{m}\right\}$ is the alphabet of $T$ and the $A_{i} \Leftrightarrow A_{i}^{\prime}$ are the defining relations. We let 1 denote the empty word. For a word $A$ on the alphabet of $T$, let $[A]_{T}$ denote the equivalence class of all words equivalent to $A$ by means of the defining relations of $T$. Thus, $[A]_{T}$ is an element of $S_{T}$, and in particular $\left\{\left[a_{i}\right] \mid 1 \leqq i \leqq m\right\}$ generates $S_{T}$ and $[1]_{T}$ is the identity element of $S_{T}$. Rather than distinguish between $a_{i}$ as a letter of the alphabet of $T$ and $\left[a_{i}\right]$ as a generator of $S_{T}$, we will use the word generator to cover both cases. When $[A]_{T}=\left[A^{\prime}\right]_{T}$ we may also write $A \sim A^{\prime}$ in $T$ to emphasize the fact that there must be a derivation of $A^{\prime}$ from $A$ ( $A$ from $A^{\prime}$ ) in $T$. Also we write $[A]=\left[A^{\prime}\right]$ in $T$ to avoid the subscript.

Received by the editors August 7, 1972.

AMS (MOS) subject classifications (1970). Primary 20M05, 02F05, $02 \mathrm{~F} 50$.

Key words and phrases. Finitely presented semigroup, free product, rank, decision problem.

(C) American Mathematical Society 1974 
Let card $[A]_{T}$ denote the number of distinct words equivalent to $A$ in $T$. We assume henceforth that no Thue system contains defining relations of the form $a_{i} \Leftrightarrow a_{j}, a_{i} \Leftrightarrow 1$, or $1 \Leftrightarrow 1$. We also assume that every semigroup mentioned in this paper is finitely presented. We write $S \simeq S^{\prime}$ for $S$ isomorphic to $S^{\prime}$. If $T_{1}$ and $T_{2}$ are Thue systems with disjoint sets of generators, then we write $T_{1} * T_{2}$ for the Thue system which has exactly the union of the two sets of generators as its generators and the union of the two sets of defining relations as its defining relations. Then $S_{T_{1}} \bullet T_{2}$ is the free product of $S_{T_{1}}$ and $S_{T_{2}}$ which is usually written $S_{T_{1}} * S_{T_{2}}$. (For more details see [C\&P, p. 140].)

Let $\mathscr{E}$ be the set of all Thue systems in which there are no defining relations of the form $1 \Leftrightarrow A$ where $A$ is a nonempty word on the generators of the Thue system. Let

$\hat{\mathscr{E}}=\{S \mid S$ is a semigroup for which there exists a $T \in \mathscr{E}$,

such that $\left.S \simeq S_{T}\right\}$.

(One may also think of $\hat{\mathscr{E}}$ as $\left\{T \mid\right.$ there exists a $T^{\prime} \in \mathscr{E}$ such that $\left.S_{T} \simeq S_{T^{\prime}}\right\}$.)

LEMMA 1. Let $T=\left\langle a_{1}, \cdots, a_{m} ; A_{i} \Leftrightarrow A_{i}^{\prime}, 1 \leqq i \leqq n\right\rangle$ be a Thue system. If $T \in \mathscr{E}$ then $\operatorname{card}[1]_{T}=1$.

Proof. If there were a nonempty word $A^{\prime}$ such that $[1]=\left[A^{\prime}\right]$ in $T$, then there would be a derivation of $1 \sim A^{\prime}$ in $T$ and that would require a defining relation of the form $1 \Leftrightarrow A$ for some nonempty word $A$.

LEMMA 2. Let $T_{1}=\left\langle b_{1}, \cdots, b_{p} ; B_{i} \Leftrightarrow B_{i}^{\prime}, 1 \leqq i \leqq q\right\rangle$ and $T_{2}=\left\langle c_{1}, \cdots, c_{r}\right.$; $\left.C_{i} \Leftrightarrow C_{i}^{\prime}, 1 \leqq i \leqq s\right\rangle$ be Thue systems in $\mathscr{E}$. Suppose $U$ and $V$ are words on the b's and c's such that $[U]=[V]$ in $S_{T_{1}} * S_{T_{2}}$. Then, if $U$ is $B_{1} C_{1} \cdots B_{k} C_{k}$ then $V$ must be $B_{1}^{\prime} C_{1}^{\prime} \cdots B_{k}^{\prime} C_{k}^{\prime}$ where, for $1 \leqq j \leqq k$, (i) $B_{j}, B_{j}^{\prime}$ are words on the b's and $C_{j}, C_{j}^{\prime}$ are words on the c's, and (ii) $\left[B_{j}\right]=\left[B_{j}^{\prime}\right]$ in $T_{1}$ and $B_{j}$ is the empty word if and only if $B_{j}^{\prime}$ is, and, $\left[C_{j}\right]=\left[C_{j}^{\prime}\right]$ in $T_{2}$ and $C_{j}$ is the empty word if and only if $C_{j}^{\prime}$ is.

Proof. Consider a derivation of $U \sim V$ in $T_{1} * T_{2}$. Since all defining relations are either from $T_{1}$ or from $T_{2}$ there is no defining relation in which both $b$ 's and $c$ 's occur on at least one side. Further, since $T_{1}, T_{2} \in \mathscr{E}$ there are no defining relations $1 \Leftrightarrow B$ or $1 \Leftrightarrow C$. So each step of a derivation takes place within a factor $B_{i}$ or a factor $C_{i}$ and cannot collapse such a factor to 1 nor insert a new factor.

Lemma 3. Let $S \simeq S_{1} * S_{2}$. Then, $S \in \hat{\mathscr{E}}$ if and only if $S_{1}$ and $S_{2} \in \hat{\mathscr{E}}$.

Proof. If $S_{1}, S_{2} \in \hat{\mathscr{E}}$ then certainly $S \in \hat{\mathscr{E}}$. So suppose $S \in \hat{\mathscr{E}}$ and let $T \in \mathscr{E}$ present $S$. If $S_{1} \notin \hat{\mathscr{E}}$, then for every Thue system which presents $S_{1}$ 
there must be some defining relation of the form $1 \Leftrightarrow B$ where $B$ is a nonempty word on the generators of that Thue system. For at least one such Thue system, say $T_{1}$, and one such relation, say $B$ is $b_{i_{1}} \cdots b_{i_{t}}$, it must be that not all $b_{i_{j}}$ are equivalent to 1 in $T_{1}$. For otherwise, we could obtain, by means of a Tietze transformation (see [MK\&S, p, 50]), a Thue system $T_{1}^{\prime}$ which presents $S_{1}$ in which none of the generators $b_{i_{1}}, \cdots, b_{i_{t}}$ occur nor does the defining relation $1 \Leftrightarrow B$; thus by repeating this process we could obtain a Thue system in $\mathscr{E}$ that presents $S_{1}$. So consider $T_{1}$ as specified. Let $\theta$ be the given isomorphism; we can consider $\theta^{-1}[1]_{T_{1}}=\theta^{-1}[B]=\theta^{-1}\left[b_{i_{1}}\right] \cdots \theta^{-1}\left[b_{i_{t}}\right]$ in $S$. For each $b_{i_{j}}, \theta^{-1}\left[b_{i_{j}}\right]=\left[A_{i_{j}}\right]$ for some word $A_{i_{j}}$ on the generators of $T$. Since there is at least one $b_{i_{j}}$ such that $\left[b_{i_{j}}\right] \neq[1]$ in $T_{1}$, there must be a nonempty $A_{i_{j}}$ such that $\theta^{-1}\left[b_{i_{j}}\right]=\left[A_{i_{j}}\right]$ in $S$. But then $[1]_{T}=\theta^{-1}[1]_{T_{1}}=\left[A_{i_{1}} \cdots A_{i_{j}} \cdots A_{i_{t}}\right]$ which contradicts the fact, from Lemma 1 , that card[1] $]_{T}=1$. Therefore, if $S \in \mathscr{E}$ and $S \simeq S_{1} * S_{2}$, then $S_{1}, S_{2} \in \hat{E}$.

For a Thue system $T$ let $|T|$ denote the number of generators of $T$. If $S$ is a semigroup let rank $S$ denote the minimal number of generators of $S$, i.e. there is a $T$ such that $S_{T} \simeq S$ and for all $T^{\prime}$ that present $S,|T| \leqq$ $\left|T^{\prime}\right|$; rank $S=|T|$.

For the semigroups in $\hat{\mathscr{E}}$ we have the following kind of analogue of Grushko's Theorem.

THEOREM 1. Suppose that $\theta: S \rightarrow S_{1} * S_{2}$ is an onto isomorphism and that $S$ or, $S_{1}$ and $S_{2}$, are in $\hat{\mathscr{E}}$. (a) Let $T=\left\langle a_{1}, \cdots, a_{m} ; A_{i} \Leftrightarrow A_{i}^{\prime}, 1 \leqq i \leqq n\right\rangle$ and suppose $T \in \mathscr{E}$ and presents $S$. If $|T|=\operatorname{rank} S$, then for each $i, 1 \leqq i \leqq m$, $\theta\left[a_{i}\right]$ is an element of $S_{1}$ or an element of $S_{2}$. Also, (b) rank $S=\operatorname{rank} S_{1}+$ rank $S_{2}$.

Proof. (a) Let $T_{1}=\left\langle b_{1}, \cdots, b_{p} ; \quad B_{i} \Leftrightarrow B_{i}^{\prime}, \quad 1 \leqq i \leqq q\right\rangle$ and $T_{2}=$ $\left\langle c_{1}, \cdots, c_{r} ; C_{i} \Leftrightarrow C_{i}^{\prime}, 1 \leqq i \leqq s\right\rangle$ present $S_{1}$ and $S_{2}$, respectively. By Lemma 3 we may assume that $T_{1}, T_{2} \in \mathscr{E}$. The proof is by contradiction. Suppose there is an $a_{i}$ such that $\theta\left[a_{i}\right] \in S_{1} * S_{2}-\left(S_{1} \cup S_{2}\right)$. Designate those $a_{i}$ for which $\theta\left[a_{i}\right] \in S_{1}$ by $a_{i}^{\prime}$, those for which $\theta\left[a_{i}\right] \in S_{2}$ by $a_{i}^{\prime \prime}$, and those for which $\theta\left[a_{i}\right] \in S_{1} * S_{2}-\left(S_{1} \cup S_{2}\right)$ by $\bar{a}_{i}$. We claim first that for each $b_{i}$, if $A_{i}$ is a word on the generators of $T$ such that $\theta^{-1}\left[b_{i}\right]=\left[A_{i}\right]$, then in fact $A_{i}$ is a word on the $a_{i}^{\prime}$ 's. (A similar claim is made for each $c_{i}$.) For, suppose there is a word $A$ of the form $A_{1} a_{j}^{\prime \prime} A_{2}$ or $A_{1} \bar{a}_{j} A_{2}$ such that $\theta^{-1}\left[b_{i}\right]=[A]$. Since $\theta\left[a_{j}^{\prime \prime}\right]=\left[C_{j}\right]$ and $\theta\left[\bar{a}_{j}\right]=\left[B_{1} C_{1} \cdots B_{t} C_{t}\right]$ where at least one $B_{i}$ and one $C_{j}$ are not the empty word, then there is a word $W$ such that $\theta \theta^{-1}\left[b_{i}\right]=\left[b_{i}\right]=[W]$ and $W$ contains both nonempty subwords on $b_{i}$ 's and nonempty subwords on $c_{i}$ 's. But, by Lemma 2 this is impossibleany $W$ such that $\left[b_{i}\right]=[W]$ must be on $b_{i}$ 's only. (A similar argument holds for the $c_{i}$.) Now consider any $\bar{a}_{i} \cdot \theta[\bar{a}]=\left[B_{1} C_{1} \cdots B_{t} C_{t}\right]$ where at 
least one $B_{i}$ and one $C_{j}$ are nonempty words on $b_{i}$ 's and $c_{i}$ 's respectively. Then, by the claim just established there are words $A_{j}^{\prime}$ on the $a_{i}^{\prime}$ 's and $A_{j}^{\prime \prime}$ on the $a_{i}^{\prime \prime \prime}$ s such that $\theta^{-1}\left[B_{j}\right]=\left[A_{j}^{\prime}\right]$ and $\theta^{-1}\left[C_{j}\right]=\left[A_{j}^{\prime \prime}\right]$ for $1 \leqq i \leqq t$. Therefore

$$
[\bar{a}]=\theta^{-1} \theta[\bar{a}]=\theta^{-1}\left[B_{1}\right] \theta^{-1}\left[C_{1}\right] \cdots \theta^{-1}\left[C_{t}\right]=\left[A_{1}^{\prime} A_{1}^{\prime \prime} \cdots A_{t}^{\prime \prime}\right] .
$$

That is, $\bar{a}$ is equivalent to a word in which $\bar{a}$ does not occur. Thus, by a Tietze transformation, there is a presentation of $S$ with one less generator, so $|T|>\operatorname{rank} S$.

For the proof of (b) suppose $T$ presents $S$ and $|T|=\operatorname{rank} S$, and $T_{i}$ presents $S_{i}$ for $i=1,2$. Suppose $T, T_{1}$ and $T_{2}$ as given by the theorem and the proof of part (a). Since $T_{1} * T_{2}$ presents $S$ and $\left|T_{1} * T_{2}\right|=\left|T_{1}\right|+\left|T_{2}\right|$, certainly rank $S \leqq\left|T_{1}\right|+\left|T_{2}\right|$. As in part (a) use $a_{i}^{\prime}$ and $a_{i}^{\prime \prime}$ to indicate which generators are sent by $\theta$ to $S_{1}$ and which to $S_{2}$. (We now know there are no $\bar{a}_{i}$.) We claim that $\left\{\theta\left[a_{i}^{\prime}\right] \mid\right.$ all $\left.a_{i}^{\prime}\right\}$ generates $S_{1}$; a similar claim is made for $S_{2}$. Let $B$ be any word on the $b_{i}$ 's. Then $\theta^{-1}[B]=[A]$ for some word $A$ of the form $a_{i_{1}}^{\prime} a_{i_{2}}^{\prime} \cdots a_{i_{n}}^{\prime}$. So $[B]=\theta\left[a_{i_{1}}^{\prime}\right] \cdots \theta\left[a_{i_{n}}^{\prime}\right]$. So there are Thue systems $T_{1}^{\prime}$ and $T_{2}^{\prime}$, which present $S_{1}$ and $S_{2}$, respectively, such that $\left|T_{1}^{\prime}\right|$ equals the number of $a_{i}^{\prime \prime}$ s and $\left|T_{2}^{\prime}\right|$ equals the number of $a_{i}^{\prime \prime \prime}$ s. So rank $S=\operatorname{rank} S_{1}+\operatorname{rank} S_{2}$. (Remark: it is easy to show that $T_{1}^{\prime}, T_{2}^{\prime} \in \mathscr{E}$.)

Corollary. If $S \in \hat{\mathscr{E}}$ and $S \simeq S * S^{\prime}$ for some semigroup $S^{\prime}$, then $S^{\prime}$ is the trivial semigroup.

Proof. By Lemma 3, $S \in \hat{\mathscr{E}}$ implies that $S^{\prime} \in \hat{\mathscr{E}}$, and by Theorem 1, rank $S=\operatorname{rank} S+\operatorname{rank} S^{\prime}$, so rank $S^{\prime}$ must be 0 .

THEOREM 2. For any given $T$ such that $S_{T} \in \hat{\mathscr{E}}$ there is no alogrithm to decide of an arbitrary Thue system $T^{\prime}$ whether or not $S_{T} \simeq S_{T^{\prime \prime}}$.

Proof. Let $T_{0} \in \mathscr{E}$ such that $S_{T_{0}} \simeq S_{T}$. Consider $\left\{S_{T_{0}} * S_{T^{\prime}} \mid T^{\prime}\right.$ varies over all Thue systems with generators

disjoint from those of $\left.T_{0}\right\}$.

Then, by the corollary, $S_{T_{0}} \simeq S_{T_{0}} * S_{T^{\prime}}$ if and only if $S_{T^{\prime}}$ is trivial. But it is known (see [M] or [Mo]) that there is no algorithm to decide of an arbitrary Thue system whether or not it presents the trivial semigroup.

These two theorems have a certain will-o'-the-wisp quality since, as proved below, $\hat{\mathscr{E}}$ is not a recursive set. A Markov property $\hat{\mathscr{P}}$ of finitely presented semigroups is a property (i.e. a class of semigroups) which is closed under isomorphism and for which there are Thue systems $T_{1}$ and $T_{2}$ such that (i) $S_{T_{1}} \in \hat{\mathscr{P}}$ and (ii) for any semigroup $S$ if $S_{T_{2}}$ can be embedded in $S$, then $S \notin \hat{\mathscr{P}}$. 
LEMMA 4. $\hat{\mathscr{E}}$ is a Markov property.

Proof. $\quad \hat{\mathscr{E}}$ obviously satisfies (i), For (ii), consider $T=\langle a, b ; a b \Leftrightarrow 1\rangle$; it does not belong to $\mathscr{E}$. We will see that $S_{T}$ cannot be embedded in any $S$ that belongs to $\hat{\mathscr{E}}$. For any $S$ and $\theta$ such that $\theta$ embeds $S_{T}$ into $S$ we have $\theta[a] \cdot \theta[b]=[1]$ in $S$. Since $[a],[b]$ and [1] are distinct elements of $S_{T}$, for any presentation $T^{\prime}$ of $S$ there must be nonempty words $A$ and $B$ on the generators of $T^{\prime}$ such that $\theta[a]=[A]$ and $\theta[b]=[B]$. Thus $A B \sim 1$ in $T^{\prime}$ so there must be some defining relation of the form $A_{i} \Leftrightarrow 1$ in $T^{\prime}$. Therefore $T^{\prime} \notin \mathscr{E}$ and so $S \notin \hat{\mathscr{E}}$.

THEOREM 3. $\quad \hat{\mathscr{E}}$ is not recursive but is recursively enumerable.

Proof. In [M] (or see [Mo]), Markov shows that for any Markov property $\hat{\mathscr{P}}$ there can be no algorithm to decide of an arbitrary Thue system $T$ whether or not $S_{T} \in \hat{\mathscr{P}}$. On the other hand, $\mathscr{E}$ is recursive, so by recursively enumerating all Thue systems presenting semigroups isomorphic to semigroups presented by Thue systems in $\mathscr{E}$ one obtains a recursive enumeration of $\hat{\mathscr{E}}$. That one can recursively enumerate the Thue systems presenting semigroups isomorphic to a given one is generally believed; a proof is offered in [Y2].

I am very grateful to Charles F. Miller III for suggesting that I think about Grushko's and Rabin's theorems for semigroups. My thanks also to the referee for some very helpful comments.

ADDED IN PROOF. Theorem 1 does not hold for semigroups in general; counterexample: $\langle a, b ; a b=1\rangle *\left\langle c ; c^{2}=1\right\rangle$. Also, let $S_{1}, S_{2}, S$ be semigroups with $S_{1} \in \hat{E}$. If $X$ is a set of generators of minimal length w.r.t. $S_{1}$ and $S_{2}$, then for each $x \in X$ either $x \in S_{1}$ or $x \in S_{2}$.

\section{REFERENCES}

[C\&P] A. H. Clifford and G. B. Preston, The algebraic theory of semi-groups, Vol. II, Math. Surveys, no. 7, Amer. Math. Soc., Providence, R.I., 1967. MR 36 \#1558.

[D] M. Davis, Computability and unsolvability, McGraw-Hill Series in Information Processing and Computers, McGraw-Hill, New York, 1958. MR 23 \#A1525.

[K] A. G. Kuroš, Theory of groups, GITTL, Moscow, 1953; English transl., Vol II, Chelsea, New York, 1960. MR 15, 501; 22 \#727.

[M] A. A. Markov, The theory of algorithms, Trudy Mat. Inst. Steklov. 42 (1954); English transl., Israel Program for Scientific Translations, Jerusalem, 1961. MR 17, 1038; 24 \#A2527.

[MK\&S] W. Magnus, A. Karrass and D. Solitar, Combinatorial group theory: Presentations of groups in terms of generators and relations, Pure and Appl. Math., vol. 13, Interscience, New York, 1966. MR 34 \#7617.

[Mo] A. Mostowski, Review of A. A. Markov's impossibility of algorithms for recognizing some properties of associative systems, J. Symbolic Logic 17 (1952), p. 151. 
[R] M. O. Rabin, Recursive unsolvability of group theoretic problems, Ann. of Math. (2) 67 (1958), 172-194. MR 22 \#1611.

[Y1] A. Yasuhara, Recursive function theory and logic, Academic Press, New York, 1971.

[Y2] — Some non-recursive classes of semi-groups with solvable word problem, Z. Math. Logik Grundlagen Math. (to appear).

Department of Computer Sciences, Rutgers University, New Brunswick, NEW JERSEY 08903 